\title{
Single photon emission computed tomography in tuberculous meningitis
}

Sanjay Gandhi Postgraduate Institute of Medical Sciences, Lucknow 226014 , India: Department of Neurology U K Misra

J Kalita

Department of Nuclear Medicine B K Das

Correspondence to: Dr Misra (email: ukmisra@sgpgi.ac.in)

Submitted 3 June 1998 Accepted 13 January 2000

\author{
U K Misra, J Kalita, B K Das
}

\begin{abstract}
Introduction-Data on single photon emission computed tomography (SPECT) in tuberculous meningitis are lacking and prompted this study. SPECT findings in tuberculous meningitis are reported and correlated with clinical and radiological findings.

Patients and methods-Seventeen patients with tuberculous meningitis that was diagnosed on clinical, radiological, and laboratory criteria have been included. Their age ranged between 5 and 62 years and four of them were female. Computed tomography and/or magnetic resonance imaging (MRI) and SPECT using ${ }^{99 \mathrm{~m}} \mathrm{Tc}-\mathrm{ethylene}$ cystine dimer were performed in all the patients. On the basis of Barthel index (BI) score the patients' outcome was defined as complete $(B I=20)$, partial $(B I=19-12)$, and poor recovery $(\mathrm{BI}<12)$.
\end{abstract}

Result -Eleven patients were in stage III and three each in stage II and stage I tuberculous meningitis. Two patients had hemiplegia and five quadriplegia. Computed tomography was abnormal in 11 out of 16 patients and revealed hydrocephalus in nine, basal exudates, infarction in subcortical white matter and basal ganglia in six patients each, frontal cortical infarction in one, and granulomata in three patients. Cranial MRI was carried out in four patients and revealed multiple granulomata, hydrocephalus, and brainstem infarction in two patients each. SPECT studies were abnormal in all except two patients and revealed hypoperfusion of the basal ganglia in 14, cortical hypoperfusion in 10, and midbrain hypoperfusion in one patient. At the three month follow up four patients had died, five had poor, three partial, and five complete recovery. The SPECT studies were more frequently abnormal compared with computed tomography but did not correlate with stage of meningitis or outcome.

Conclusion-In tuberculous meningitis subcortical and cortical hypoperfusion is common but it does not correlate with stage of meningitis or three month outcome.

(Postgrad Med f 2000;76:642-645)

Keywords: tuberculous meningitis; single photon emission computed tomography (SPECT); computed tomography; magnetic resonance imaging

Tuberculous meningitis is commonly associated with vasculitis. The frequency of vasculitis in tuberculous meningitis may depend on the method of evaluation. In an autopsy study on 100 patients, infarction was found in $41 \% .{ }^{1}$ On computed tomography, the frequency of infarction in tuberculous meningitis has ranged between $20.5 \%$ and $38 \% .^{2-4}$ Cerebral angiography has shown abnormalities mainly in the supraclinoid portion of the internal carotid artery in $14 \%$ patients, although the angiograms were abnormal in $29 \% .^{5}$ Magnetic resonance imaging (MRI) with MR angiography in tuberculous meningitis has revealed abnormalities in $55 \% .{ }^{6}$ In tuberculous meningitis, the vasculitis is most prominent in the penetrating vessels of carotid territory, which results in the infarction of deep white matter. The infarction in the areas supplied by major cortical vessels and vertebrobasilar territory, however, have been reported to be rare. ${ }^{7}$ The cerebral blood flow changes may be important in determining the clinical picture and the outcome of tuberculous meningitis. There are no reports on single photon emission computed tomography (SPECT) studies in the available literature. In this paper, we report preliminary results of a prospective study on cerebral blood flow changes in tuberculous meningitis and correlate these with clinical and radiological findings.

\section{Patients and methods}

Seventeen patients with highly probable tuberculous meningitis have been included in this study. The diagnosis was based on clinical, computed tomographic, and cerebrospinal fluid (CSF) criteria. The clinical criteria included fever, headache, and neck stiffness for more than two weeks. Supporting evidence was obtained from CSF cells $0.2 \times 10^{9} / 1$ or more with lymphocytic predominance, protein more than $1 \mathrm{~g} / \mathrm{l}$; sterile bacterial and fungal culture; computed tomography findings of hydrocephalus and exudates; and evidence of tuberculosis outside the central nervous system. The diagnostic categories included highly probable: clinical and three supportive criteria; probable: clinical and two supportive criteria; and possible: clinical and one supportive criteria. ${ }^{8}$ All the patients included in this study were highly probable cases of tuberculous meningitis. The patients were subjected to a detailed clinical examination. The severity of meningitis was graded as stage I: meningitis only; stage II: meningitis with neurological signs; and stage III: meningitis, neurological signs, and altered sensorium. 'The patients' outcome was defined on the basis of the three month Barthel index (BI) score as complete $(\mathrm{BI}=20)$, partial $(\mathrm{BI}=$ 19-12), and poor $(\mathrm{BI}<12){ }^{10}$

Cranial computed tomography was performed using a third generation scanner (W400 Hitachi, Japan). Unenhanced and postcontrast scans were obtained taking $10 \mathrm{~mm}$ 
Table 1 SPECT and computed tomography/MRI changes in tuberculosis meningitis

\begin{tabular}{|c|c|c|c|c|c|}
\hline No & Age/sex & Stage & SPECT & Computed tomography and MRI findings & Outcome (BI score) \\
\hline 1 & $62 \mathrm{M}$ & III & RBG and frontoparietal & Right frontal and subcortical infarction & Complete(20) \\
\hline 2 & $8 \mathrm{M}$ & III & BLBG, right frontal & $\begin{array}{l}\text { Communicating hydrocephalus, thalamic } \\
\text { infarction }\end{array}$ & Poor $(0)$ \\
\hline 3 & $20 \mathrm{~F}$ & III & Left frontotemporoparietal & $\begin{array}{l}\text { Hydrocephalus, bilateral subcortical } \\
\text { infarction left }>\text { right }\end{array}$ & $\operatorname{Poor}(0)$ \\
\hline 4 & $5 \mathrm{M}$ & III & BLBG, left frontal & Communicating hydrocephalus & Poor $(9)$ \\
\hline 5 & $9 M$ & III & BLBG, right temporoparietal & Hydrocephalus, tuberculoma ${ }^{\star}$ & Complete (20) \\
\hline 6 & $24 \mathrm{~F}$ & I & LBG, left temporoparietal & Communicating hydrocephalus & Partial(18) \\
\hline 7 & $11 \mathrm{M}$ & III & RBG, right parieto-occipital & Hydrocephalus, tuberculoma & Poor $(0)$ \\
\hline 8 & $15 \mathrm{M}$ & II & BLBG & Miliary granuloma ${ }^{\star}$ & Complete(20) \\
\hline 9 & $14 \mathrm{M}$ & I & Normal & Normal & Complete (20) \\
\hline 10 & $10 \mathrm{M}$ & III & BLBG and frontal & Hydrocephalus, subcortical infarction & Expired \\
\hline 11 & $24 \mathrm{M}$ & $\mathrm{I}$ & LBG and parietal & Normal & Complete(20) \\
\hline 12 & $30 \mathrm{M}$ & III & RBG & Hydrocephalus, infarction RBG & Expired \\
\hline 13 & $25 \mathrm{M}$ & III & Normal & Pontomedullary infarction ${ }^{\star}$ & Partial(15) \\
\hline 14 & $11 \mathrm{M}$ & III & $\begin{array}{l}\text { BLBG, left } \\
\text { frontotemporoparietal }\end{array}$ & RBG infarction & Expired \\
\hline 15 & $23 \mathrm{~F}$ & II & BLBG, midbrain & Hydrocephalus, left midbrain infarction ${ }^{\star}$ & $\operatorname{Partial}(18)$ \\
\hline 16 & $8 M$ & III & BLBG & Hydrocephalus, granuloma & Poor(1) \\
\hline 17 & $20 \mathrm{~F}$ & II & BLBG & Normal & Expired \\
\hline
\end{tabular}

$\mathrm{BLBG}=$ bilateral basal ganglia; $\mathrm{LBG}=$ left basal ganglia; $\mathrm{RBG}=$ right basal ganglia.

sections parallel to orbitomeatal line. Cranial MRI was performed on a $2 \mathrm{~T}$ scanner operating at $1.5 \mathrm{~T}$ (Magnetom, SP Siemens Germany). T1 (500/15/3-TR in ms/TE in ms/excitations), proton density (2000-2500/15-20/1), and T2 (2000-2500/80-90/1) weighted spin echo sequences were obtained.

SPECT studies were performed using a multidetector multichannel dedicated gamma camera (head tome set 301, Shimadzu Corporation, Kyoto, Japan) using ${ }^{99 \mathrm{~m}} \mathrm{Tc}$-ethylene cystine dimer (ECD) for the assessment of regional cerebral blood flow. The patient was positioned in the gantry and $20 \mathrm{mci}$ of ${ }^{99 m}$ Tc-ECD was injected intravenously into an antecubital vein. Subsequently cotton pads were placed on the eyes and patient was made to lie down for 30 minutes after which the data acquisition was started. The head was strapped in the headrest and light indicator system was switched on which the canthomeatal line was marked. Three rims of high resolution detectors containing 64 sodium iodide detectors obtained simultaneous slices of the brain. Tomographic images were obtained in static mode using a high resolution collimator system. The collimator rotated $360^{\circ}$ and the detector rims were fixed. Transaxial section were obtained. For image reconstructions Butterworth filters were used and slice thickness was $11 \mathrm{~mm}$. The window setting was kept at $140 \pm 20 \% \mathrm{KeV}$ and a sampling time of 30 s. An online computer coupled with the system received the data and reconstructed the images. The SPECT images were visually interpreted independently by a neurophysician experienced in SPECT studies and a nuclear medicine consultant who were unaware of the clinical and computed tomography or MRI findings. The interpretation of SPECT studies in tuberculous meningitis were based on seven healthy volunteers who underwent ${ }^{99 \mathrm{~m}} \mathrm{Tc}-\mathrm{ECD}$ SPECT study employing a similar protocol. The SPECT changes were analysed and correlated with computed tomography/MRI and clinical findings.

\section{Results}

Our results are based on 17 patients with tuberculous meningitis (see table 1). Their mean age was 18.8 (range 5-62) years and four of them were female. Eleven patients were in stage III and three each belonged to stage II and stage I meningitis. The mean Glasgow coma scale score was 11 (range 4-15). Six patients had a history of tonic clonic seizures. Hemiplegia was present in two and quadriplegia in five patients. Cranial nerve palsy was present in 11 patients and included optic atrophy in two, external ophthalmoplegia in eight and IX and X cranial nerve palsy in one patient; the latter had a lateral medullary infarction. Two patients had miliary shadows on their chest radiograph.

The CSF was abnormal in all the patients. Acid-fast bacillus was present in the CSF in one patient. Cranial computed tomography was performed in 16 patients and was abnor$\mathrm{mal}$ in 11 . The abnormalities included hydrocephalus in nine and subcortical white matter and basal ganglia infarcts in six patients (figs 1 and 3). One patient had right frontal cortical infarct. Ring enhancing lesions were present in

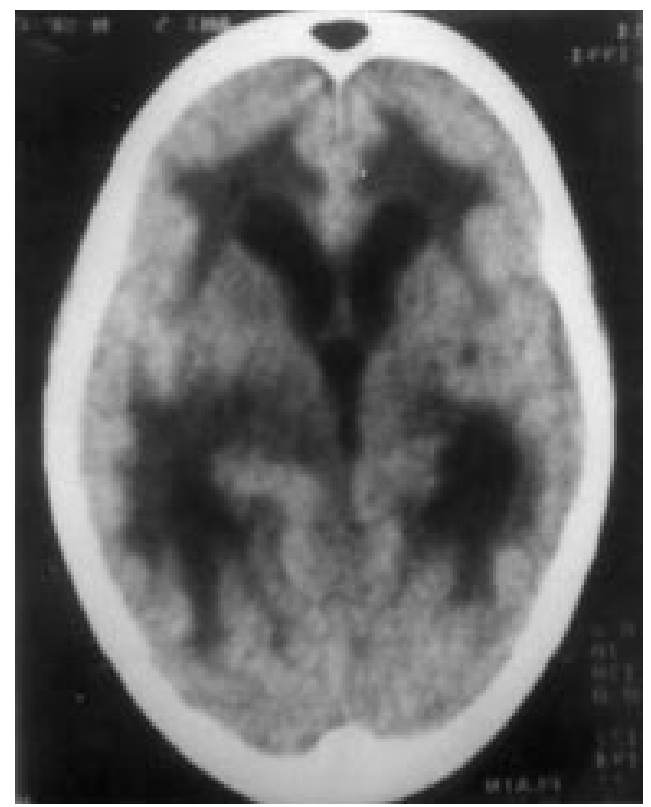

Figure 1 Computed tomography of a patient with stage III tuberculous meningitis (No 3) shows hydrocephalus and bilateral basal ganglia infarction. 


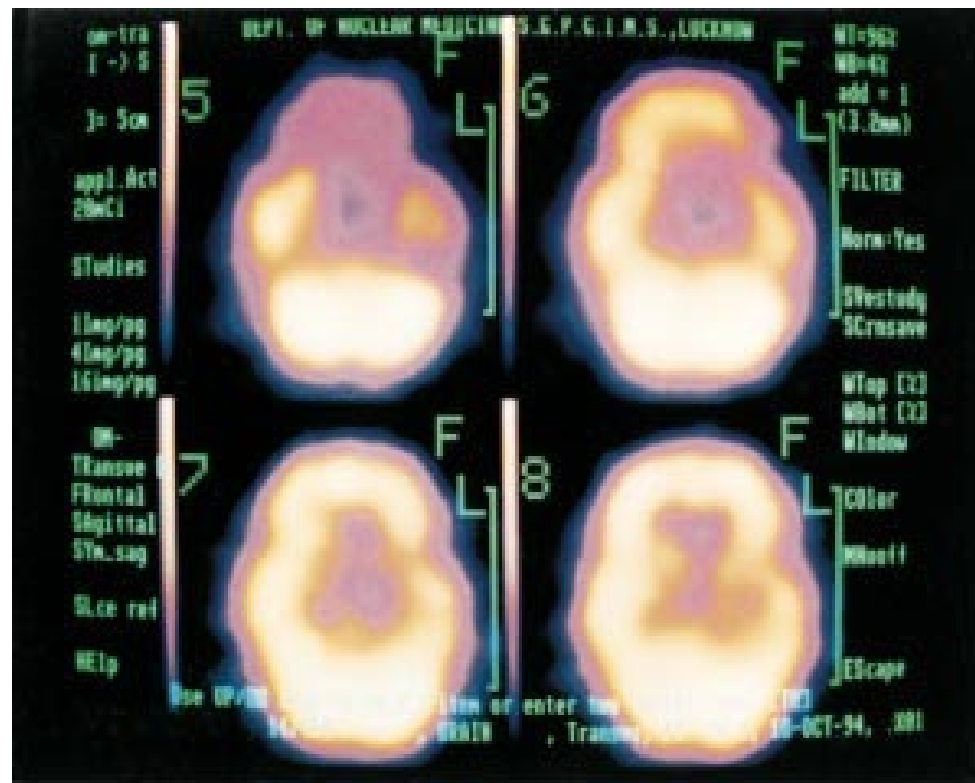

Figure 2 SPECT study of patient No 3 showing subcortical and cortical hypoperfusion in left frontotemporoparietal region.

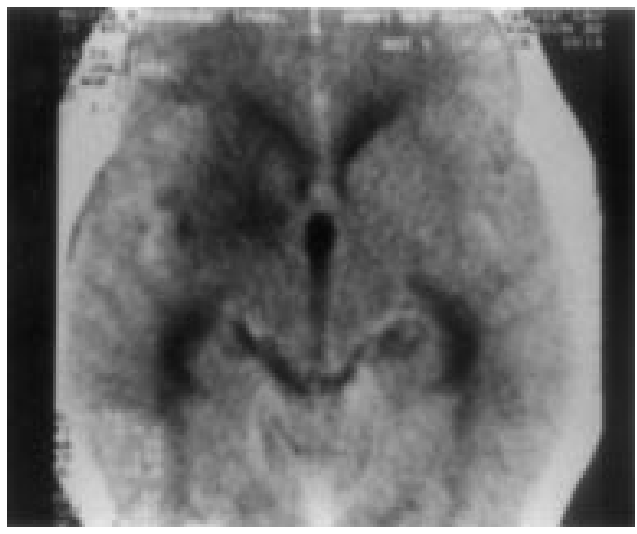

Figure 3 Computed tomography of a patient with stage III tuberculous meningitis (No 14) showing right basal ganglia infarction.

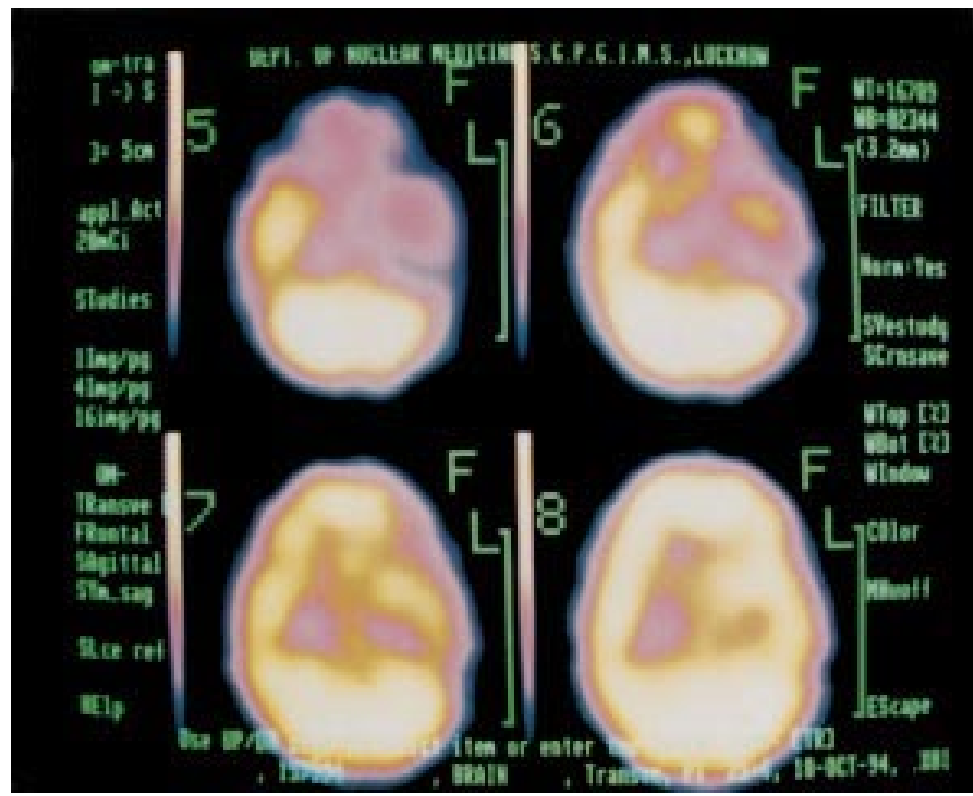

Figure 4 SPECT study of patient No 14 revealed bilateral basal ganglia and left frontotemporoparietal hypoperfusion. three and basal exudates in seven patients. MRI was performed in four patients including a patient in whom computed tomography was not possible. In three patients who had normal computed tomography, MRI revealed miliary lesions which were hyperintense on T2 and proton density, and hypointense to isointense on $\mathrm{T} 1$ in one patient. This patient also had miliary shadows on the chest radiograph. Another patient had a right lateral pontomedullary infarct. The third patient had communicating hydrocephalus and a left midbrain infarct. The fourth revealed communicating hydrocephalus, exudates, and multiple intracranial granulomatas.

SPECT

The SPECT studies were abnormal in all except two patients. Basal ganglia hypoperfusion was present in 14 patients, which was bilateral in nine. Cortical hypoperfusion was present in 10 patients which involved frontal region in three, parietal in one and frontoparietal in one, parieto-occipital in one, frontotemporoparietal in two, and temporoparietal in two patients (figs 2 and 4). Basal ganglia hypoperfusion correlated with a corresponding infarct on computed tomography or MRI in five patients. Out of 10 patients with cortical hypoperfusion, infarct on the computed tomogram was present in one patient only. The patient with midbrain hypoperfusion did have a midbrain infarct on MRI.

There were two patients with basal ganglia hypoperfusion without evidence of infarct on computed tomography or MRI. All the nine patients with hydrocephalus had SPECT abnormalities, which included basal ganglia hypoperfusion in all and focal cortical hypoperfusion in six patients.

At the three month follow up, four patients had died, five had poor, three partial, and five complete recovery. SPECT did not correlate with the severity of meningitis $\left(\chi^{2}=1.82, \mathrm{df}=2\right.$, not significant) because out of two patients with normal SPECT, one had stage I and the other stage III meningitis. Of the 14 patients with abnormal SPECT, two patients had stage I, three stage II, and 10 stage III meningitis. The blood flow changes also did not seem to correlate with the outcome $\left(\chi^{2}=0.08, \mathrm{df}=1\right.$, not significant). Out of five patients who recovered completely, four had basal ganglia and three cortical hypoperfusion. The four patients who died all had basal ganglia hypoperfusion and cortical hypoperfusion was present in two.

\section{Discussion}

The results of the present study reveal three patterns of regional blood flow in tuberculous menigitis:

(1) Basal ganglia hypoperfusion

(2) Basal ganglia with cortical hypoperfusion

(3) Normal SPECT

All the patients with basal ganglia infarct on computed tomography or MRI had hypoperfusion in the corresponding area on SPECT. Infarction in tuberculous menigitis may be due 
to vasculitis or mechanical strangulation of the vessels by the surrounding exudates. The vascular pathology includes simple inflammatory infiltration, verrucus subendothelial protrusion with or without tubercle formation, concentric subintimal proliferation with narrowing of lumen, and intimal fibrinoid degeneration. ${ }^{7}$ The frequency of infarction in tuberculous menigitis varies from $10.5 \%$ to $38 \%$ in radiological studies. ${ }^{2411}$ The majority of infarctions in tuberculous menigitis are located in basal ganglia and internal capsules and are uncommon in brainstem and major vascular territories. ${ }^{211}$ In our study also only two patients had brainstem infarct nd the patient with midbrain infarct revealed hypoperfusion in the midbrain region. The SPECT study in the patient with pontomedullary infarction, however, was normal.

The SPECT studies revealed hypoperfusion in basal ganglia in nine patients who did not have infarcts in the corresponding area on computed tomography or MRI. In these patients, the basal ganglia hypoperfusion may be due to subtle blood flow changes which may not result in infarct on computed tomography or MRI. This may also be due to additional pathologies such as hydrocephalus or tuberculoma, which are common in tuberculous menigitis. There were nine patients who had both basal ganglia and cortical hypoperfusion. On computed tomography, cortical infarction was present in one and subcortical in three patients. Cortical hypoperfusion due to a subcortical infarction has been reported in stroke patients. ${ }^{12-14}$ Cerebral diaschisis is attributed to anterograde wallerian degeneration, retrograde degeneration, trans-synaptic degeneration of cortical neurons, or reduced functional activity of cortical neurons without actual degeneration. ${ }^{13}$ The latter mechanisms seems to be more likely in our patients. Moreover, in our patients hypoperfusion without any infarction in the computed tomogram could be due to raised intracranial pressure and accompanying hydrocephalus. In the available literature, we could not find any SPECT studies in tuberculous meningitis, however, in acute hydrocephalus after subarachnoid haemorrhage decreased regional cerebral blood flow was found in the basal part of brain, around the third ventricle, temporal horn of lateral ventricle, and frontal lobes. ${ }^{15}$ In hydrocephalus regional cerebral blood flow improved after shunt surgery. ${ }^{16}$ In normal pressure hydrocephalus, although no characteristic SPECT pattern has been reported, bifrontal hypoperfusion is a common finding. ${ }^{17}$ It is possible that after hydrocephalus there may be distortion of large feeding vessels and collapse of capallaries, which may account for reduced regional cerebral blood flow. ${ }^{16}$ In our study, nine patients had hydrocephalus, and SPECT abnormalities included basal ganglia hypoperfusion in all and cortical hypoperfusion in six.
In the setting of tuberculous meningitis with vasculitis this effect may be more apparent in the compromised vascular territory. A comparison of SPECT results with cerebral angiography, however, could have provided better understanding of the mechanisms of hypoperfusion in tuberculous meningitis.

In our study, SPECT revealed more frequent abnormalities compared with computed tomography. The SPECT abnormalities show the functional alterations, whereas computed tomography provides information about structural changes. These studies are complementary and not competitive. The SPECT changes neither related with stage of tuberculous meningitis nor with outcome. The lack of correlation may be due to small number of mildly affected patients in our study.

It can be concluded from this study that in tuberculous meningitis, subcortical and cortical hypoperfusion is common but it does not correlate with the severity of meningitis or with three month outcome. A larger study, preferably with sequential follow up, would provide more definite answers.

We gratefully acknowledge the financial grant from Department of Science and Technology, India (project No SP/SO/No5/94) and the help of Mr Rakesh Kumar Nigam for preparing the manuscript.

1 Dastur DK, Lalitha VS, Udani PM, et al. The brain and meninges in the gross pathology in 100 cases and pathogenesis. Neurology (India) 1970;18:86-100.

2 Bhargava S, Gupta AK, Tandon PN. Tuberculous meningitis—a CT study. Br F Radiol 1982;55:189-96.

3 Bonafe A, Manilfe C, Gomez MC, et al. Tuberculous meningitis. Contribution of computerized tomography to its diagnosis and prognosis. I Neuroradiol 1985;12:302-16.

4 Leiguarda R, Berthier M, Starkstein S, et al. Ischaemic infarction in 25 children with tuberculous meningitis. Stroke 1988;19:200-4.

5 Dalal PM. Observations on the involvement of cerebral vessels in tuberculous meningitis in adults. In: Goldstein M, ed. sels in tuberculous meningitis in adults. In: Goldstein M, ed.
Advances in neurology. Vol 25. New York: Raven Press, 1979: 149-59

6 Gupta RK, Gupta S, Singh D, et al. MR imaging and angiography in tuberculous meningitis. Neuroradiology 1994;36: 87-92.

7 Dalal PM, Dalal KP. Cerebrovascular manifestations of infectious disease. In: Toole JF, ed. Handbook of clinical neurology. Vol II (55): Vascular disease part III. Amsterdam: Elsevier 1989: 411-41.

8 Shankar P, Manjunath N, Mohan KK, et al. Rapid diagnosis of tuberculous meningitis by polymerase chain reaction. Lancet 1991;337:5-7.

9 Lincoln EM, Sordillo SVR, Davics PA. Tuberculous meningitis in children. $\mathcal{F}$ Pediatr 1960;57:807-10.

10 Misra UK, Kalita J. Putaminal haemorrhage leading to pure motor hemiplegia. Acta Neurol Scand 1995;91:283-6.

11 Jinkins JR. Computed tomography of intracranial tuberculosis. Neuroradiology 1991;33:126-36.

12 Tanaka M, Kondo S, Hirai S, et al. Cross cerebellar diaschisis accompanied by hemitaxia: PET study. $\mathcal{F}$ Neurol Neurosurg Psychiatry 1992;52:121-5.

13 Baron JC, D'Antona RD, Pantono P, et al. Effects of thalamic stroke on energy metabolism of cerebral cortex: a positron emission tomography study in man. Brain 1986; 109:1243-59.

14 Pantano P, Baron JC, Samson Y, et al. Crossed cerebellar diaschisis further studies. Brain 1986;109:677-94.

15 Hasen D, Van-Peski J, Loeve I, et al. Single photon emission computed tomography in patients with acute hydrocephalus or with cerebral ischaemia after subarechnoid haemorrhage. f Neurol Neurosurg Psychiatry 1991;54:492-3.

16 Caner H, Caner B, Turul H, et al. Pre and postoperative assessment of regional cerebral blood flow in hydrocephalus by $99 \mathrm{mTc}$ hexamethylpropylenamine oxime SPECT. $f \mathrm{Nucl}$ Biol Med 1991;35:66-72.

17 Mathew NT, Meyer JS, Hartman A, et al. Abnormal cerebral blood flow dynamics: implication in diagnosis treatment and prognosis of normal pressure hydrocephalus. Arch Neurol 1975;32:657-64. 